\title{
Incommensurability and Evidence
}

\author{
By: Jed Buchwald
}

Incommensurability between successive scientific theories -- the impossibility of empirical evidence dictating the choice between them -- was Thomas Kuhn's most controversial proposal. Toward defending it, he directed much effort over his last 30 years into formulating precise conditions under which two theories would be undeniably incommensurable with one another. His first step, in the late 1960s, was to argue that incommensurability must result when two theories involve incompatible taxonomies. The problem he then struggled with, never obtaining a solution that he found entirely satisfactory, was how to extend this initial line of thought to sciences like physics in which taxonomy is not so transparently dominant as it is, for example, in chemistry. We will reconsider incommensurability in the light of examples in which evidence historically did and did not carry over continuously from old laws and theories to new ones. The transition from ray to wave optics early in the nineteenth century, we argue, is especially informative in this regard. The evidence for the theory of polarization within ray optics did not carry over to wave optics, so that this transition can be regarded as a prototypical case of discontinuity of evidence, and hence of incommensurability in the way Kuhn wanted. Yet the evidence for classic geometric optics did carry over to wave optics, notwithstanding the fundamental conceptual readjustment that Fresnel's wave theory required. 